\title{
Integration of Spatial Relations across Perceptual Experiences
}

\author{
Marios N. Avraamides ${ }^{1}$, Christina Adamou ${ }^{1}$, Alexia Galati ${ }^{1}$, and Jonathan W. Kelly ${ }^{2}$ \\ ${ }^{1}$ Department of Psychology, University of Cyprus \\ P.O. Box 20537, 1678 Nicosia, Cyprus \\ ${ }^{2}$ Department of Psychology, Iowa State University \\ W112 Lagomarcino Hall, Ames, IA 50010 \\ \{mariosav, adamou.d.christina, galati\}@ucy.ac.cy, \\ jonkelly@iastate.edu
}

\begin{abstract}
People often carry out tasks that entail coordinating spatial information encoded in temporally and/or spatially distinct perceptual experiences. Much research has been conducted to determine whether such spatial information is integrated into a single spatial representation or whether it is kept in separate representations that can be related at the time of retrieval. Here, we review the existing literature on the integration of spatial information and present results from a new experiment aimed at examining whether locations encoded from different perspectives in the same physical environments are integrated into a single spatial representation. Overall, our findings, coupled with those from other studies, suggest that separate spatial representations are maintained in memory.
\end{abstract}

Keywords: Integration of spatial information, Reference frames, Spatial memory organization, Perspective taking.

\section{Introduction}

Much of our everyday activity relies on retrieving spatial information from memory. For example, when planning a route prior to navigating a familiar environment we typically consider where the goal location is relative to our starting point, but also how landmarks along the route relate spatially to each other. Also, during navigation we must monitor our orientation by determining where we are in relation to immediate and distal landmarks. To carry out such tasks effectively, we must construct accurate spatial representations when we experience the space (e.g., when navigating a city for the first time) and maintain those representations in memory. Whereas in some cases people construct spatial representations by experiencing multiple locations simultaneously or near simultaneously from a fixed standpoint (e.g., when looking at a small room from its entrance, or when inspecting a table-top arrangement of objects from a specific direction), in other cases, they do so by experiencing locations at different times and typically from different standpoints (e.g., viewing objects by moving within a multi-room house). 
Previous research has established that people can successfully compute the relations between locations acquired through distinct experiences (e.g., they can point to unseen distal landmarks with above chance accuracy). However, what is not yet clear is whether people integrate spatial information experienced at different points in time into a single spatial representation or maintain it in distinct representations that can be related at task execution. In the present paper we review literature that can shed light on this issue. In section 1, we provide a brief introduction on how people organize in spatial memory information experienced simultaneously (or nearsimultaneously). This introduction highlights that objects that can be viewed at once, typically as part of a layout that is external to the observer, are stored in a single representation maintained in memory from a preferred direction. Next, in section 2 , we present evidence that locations encoded sequentially within the same spatial environment are also remembered from a preferred direction, which suggests that they are integrated into a single representation. Finally, in subsequent sections we discuss the results from studies that have examined, using different paradigms, spatial memory for temporally and/or spatially separated layouts. Findings from these studies generally suggest that spatial information is not integrated into a single representation, although often experiencing one layout may influence the way objects in subsequent layouts are encoded.

\section{Memory for Locations Viewed Simultaneously}

Mounting evidence suggests that locations in spatial layouts are encoded on the basis of allocentric reference frames that are maintained in memory in a preferred direction (McNamara, 2003; Mou \& McNamara, 2002). Such evidence comes from studies that examine the organizational structure of spatial memories by having participants study a layout from an external standpoint and then, in a different laboratory room, make Judgments of Relative Direction (JRD); that is, respond to statements of the form "Imagine standing at $\mathrm{x}$ facing $\mathrm{y}$, point to $\mathrm{z}$ ", where $\mathrm{x}, \mathrm{y}$, and $\mathrm{z}$ are objects from the memorized layout. These studies generally show that pointing performance is faster and/or more accurate from one or more imagined perspectives. This is typically interpreted as evidence that during learning, participants created a spatial representation that was stored in memory from a particular orientation, axis, or set of axes (see McNamara, 2003 for a review).

Many studies in this area have focused on identifying the factors that determine the preferred direction(s) from which spatial memories are maintained. For example, environmental cues play an important role in selecting a preferred direction in memory (Shelton \& McNamara, 2001). In this study, participants studied a layout of 7 objects placed on a square mat within a rectangular room. In one experiment the edges of the mat were aligned with the walls of the room and participants viewed the objects from two standpoints: one that was aligned with the mat and the walls of the room $\left(0^{\circ}\right)$ and one that was not $\left(135^{\circ}\right)$. Viewing order was counterbalanced across participants. Subsequent JRD testing revealed that, for both viewing orders, performance was better when responding from the aligned $0^{\circ}$ than from the misaligned $135^{\circ}$ perspective, 
which was no better than the remaining non-experienced perspectives. That participants used the array's alignment with respect to the room over their own misaligned orientation as an organizing axis, highlights how powerful environmental cues are when selecting a preferred direction in memory. Similarly, other studies have provided evidence that other cues available during learning may determine the preferred direction. These cues include the presence of an axis of bilateral symmetry (Mou, Zhao, \& McNamara, 2007), instructions (Greenauer \& Waller, 2008), and other things being equal, egocentric experience (Shelton \& McNamara, 1997).

In summary, research with scenes external to the observer indicates that spatial information is maintained in memory from a preferred direction that is selected during learning based on available cues. This suggests that if locations encoded in distinct experiences are integrated into a single spatial representation, this representation should have a preferred direction observable in subsequent testing. In the next section we discuss whether locations encoded sequentially are indeed maintained in a single representation.

\section{Memory for Locations Viewed Sequentially}

In contrast to studies with table-top displays and other layouts that can be viewed at once, studies have also examined spatial memories for room-size environments in which not all objects can be viewed simultaneously. A typical set-up involves objects that are placed around the observer at different angles (e.g., Hodgson \& Waller, 2006; Kelly, Avraamides, \& Loomis, 2007). Being positioned within or internal to the layout, the observer must thus move her head or body in order to inspect all locations. Despite this additional requirement, which results in processing locations sequentially, to the best of our knowledge, no study has reported any differences in the organizational structure of memories in which the observer's position is interval vs. external to the layout.

In fact, people's memories for scenes viewed sequentially, while being internal to the scene, seem to be organized around the same principles as their memories for table-top scenes and scenes viewed at once. For example, Kelly et al. (2007) extended findings regarding the organization of memories for externally viewed scenes to scenes viewed internally, within a virtual-reality environment. In this study, participants learned the locations of 8 objects placed in the corners of an octagonal virtual room. All participants began inspecting objects from the same orientation but were then allowed to freely rotate and study the layout from any orientation and for as long as they wanted. Due to the narrow horizontal field of view of the Head-MountedDisplay (HMD) that was used, no more than 2 objects could be viewed simultaneously from any orientation, ensuring their sequential viewing. Following learning, participants were tested using JRD while standing in either the same room in which learning took place or in a different room, and while assuming an orientation that was offset by $90^{\circ}$ to the left or right of the learning orientation. Participants tested in a different room exhibited superior performance when pointing to objects from an imagined orientation that was aligned with the initial orientation they had during 
learning. This result replicates previous findings from studies with external scenes showing that in the absence of other cues or instructions, egocentric experience is used to determine a preferred direction in memory (Shelton \& McNamara, 1997). In addition, those tested in the same room also did well when they responded from an orientation that was aligned to their actual testing orientation. This advantage for people's actual orientation at the time of retrieval has also been documented with external layouts (Mou, McNamara, Valiquette, \& Rump, 2004).

The parallel results from studies with internal vs. external layouts suggest that people can easily integrate into a single spatial representation locations that are encoded sequentially. This conclusion is further corroborated by the findings of a study that manipulated the temporal presentation of to-be-learned targets. Avraamides, Loomis, Klatzky, and Golledge (2004) asked participants to indicate the relative direction and distance between pairs of objects that they had previously encoded by vision or spatial language. For our purposes, the experiments where objects were encoded through vision are pertinent. In one experiment, four visual targets were presented simultaneously in the frontal visual field of participants, whereas in a second experiment, the same targets were presented sequentially and in isolation (i.e., the previous object was removed before a new one was placed). Regardless of how participants had encoded visual targets across the two experiments, both their response latency and the standard deviation of their signed pointing errors were equivalent.

Overall, results from studies in which the encoding of spatial locations occurs incrementally within the boundaries of the same physical space suggest that people have no difficulty integrating information within a single spatial representation. In the next section, we discuss studies that have examined whether people integrate spatial locations encoded with greater temporal separation.

\section{Integration of Layouts with Extended Temporal Separation}

As our review so far suggests, when people encode spatial locations sequentially (typically as observers internal to the scene) they easily integrate into a single representation. However, it is unclear whether they also do so when the temporal separation between locations is greater than the time needed to turn their head to view an object. Do they keep these locations in distinct representations or do they integrate them in a single representation?

Studies with large-scale environments provide converging evidence that these environments are also represented in memory from preferred directions. For example, Werner and Schmidt (1999) showed that people in Göttingen, Germany pointed faster and more accurately to landmarks in their city when imagining themselves at orientations that were aligned than misaligned with the two streets of a main intersection. This suggests that their spatial memory was maintained from preferred directions that were determined by the structure of the environment. Findings from McNamara, Rump, and Werner (2002) corroborate further this conclusion. In this study participants navigated a park following one of two paths. One path was aligned 
with the intrinsic axes of a salient landmark (i.e., a Parthenon replica) and the other one was misaligned. Then, while in the lab, they pointed towards various park objects from imagined perspectives. Results revealed that participants walking the aligned path pointed more accurately from (1) perspectives aligned with the legs of the path and the intrinsic axes of the Parthenon, and (2) a perspective oriented towards a second salient landmark (i.e., a lake). Those walking the misaligned path pointed more accurately from the perspective oriented towards the lake, with accuracy for the remaining perspectives decreasing with increasing angular disparity from that perspective. Thus, in both cases participants organized their memories on the basis of a reference frame that was intrinsic to the layout, with the preferred direction being influenced by the alignment of the path.

At this point, it should be noted that a small number of studies have provided evidence that spatial reasoning about large-scale or even room-size navigable environments is orientation-independent (e.g., Evans \& Pezdek, 1980; Presson, DeLange, \& Hazelrigg, 1987; 1989). For example, in one experiment, Evans and Pezdek had college students judge the depicted spatial relations of triads containing either campus landmarks or American States, with the triads shown at various orientations. When participants judged relations among triads of States, their response latency increased linearly as a function of the angular deviation of the triad from the upright orientation typically shown in a map. However, when judging campus landmarks, which were presumably encoded in memory through active exploration as opposed to observing a map, this wasn't the case: participants judged the campus triads equally fast from every presented orientation. Although this finding may suggest that the spatial representation containing campus landmarks was orientationfree, an alternative possibility is that students had experienced campus landmarks from various orientations and constructed a representation with multiple preferred directions. But there is evidence that even unfamiliar environments may be represented in orientation-independent representations, as suggested by participants' memory performance after walking long paths in the laboratory (Presson, DeLange, \& Hazelrigg, 1987; 1989). However, subsequent studies have failed to replicate such orientation-independence for unfamiliar environments, suggesting that they may be limited to the specific testing situations employed by Presson and colleagues (see Roskos-Ewoldsen, McNamara, Shelton, \& Carr, 1998; Waller, Montello, Richardson, \& Hegarty, 2002). In general, the majority of studies on both outdoor and indoor navigable environments suggest that the constructed memories for these environments are orientation-dependent, just like the memories for locations that are experienced either simultaneously or in close temporal proximity.

A number of studies in which participants experience locations with extended temporal separation allow for further insight into whether the resulting representations are orientation-dependent and whether they involve the integration of locations. In the following subsections we review relevant findings from such studies using different paradigms: studies examining memories for nested environments, studies assessing integration by comparing responses for within- and between-layout judgments, and studies using the transfer of reference frames paradigm. 


\subsection{Integration Assessed across Nested Environments}

One approach to examining whether people integrate locations they have encoded with temporal separation is to evaluate their representations of locations in nested environments. Wang and Brockmole (2003a, 2003b) did precisely that by having people reason both about a newly learned local environment (e.g., the research laboratory) and a familiar, large-scale environment in which the new environment was nested (e.g., the campus in which the laboratory is located).

In one study (Wang \& Brockmole, 2003a), they examined whether participants arriving at the laboratory would integrate new knowledge about the locations of laboratory objects into their existing spatial representation of the campus. In a first experiment, participants were exposed to a number of laboratory objects and following a brief rotation, they pointed towards both laboratory objects and campus landmarks. Participants made larger configuration errors ${ }^{1}$ when pointing to campus landmarks than laboratory objects, suggesting that they held objects from the two environments in distinct representations. Moreover, while heading error ${ }^{2}$ was uniformly distributed for laboratory objects, it was randomly distributed for campus landmarks. This suggests that participants remained oriented within the laboratory but failed to relate their orientation to the more distal campus landmarks. In a follow-up experiment, participants walked a route from the laboratory to the campus and back, and pointed towards objects and landmarks along the way. Participants could point correctly to the direction of a campus landmark only when they exited the room. Conversely, once they were on campus grounds they lost track of their orientation relative to the room layout. These findings indicate the newly acquired spatial knowledge for a local layout is not readily integrated during learning into an existing representation of a larger scale environment. Rather, separate representations are maintained. Although this is consistent with accounts of hierarchical representations of space (Hirtle \& Jonides, 1985), it is evident that in this study participants did not represent in memory the directional relation between the two spatial representations.

In another study, Wang and Brockmole (2003b) investigated whether people automatically update spatial relations in one environment when rotating with respect to the objects of another environment. Participants, sitting on a swivel chair, learned the locations of laboratory objects and brought to mind the locations of familiar campus landmarks. Then they were asked to physically rotate to various orientations relative to either laboratory objects or campus landmarks, depending on the condition. At the end of a series of rotations they pointed to both laboratory objects and campus landmarks. When participants turned relative to laboratory objects, they were faster to point to these objects than to campus landmarks. In contrast, when they rotated relative to campus landmarks they were equally fast at pointing to campus landmarks and laboratory objects. These findings indicate that participants held laboratory

\footnotetext{
${ }^{1}$ Configuration Error is the standard deviation of the signed pointing errors. It is a measure of the internal consistency of the spatial representation, i.e., how accurate an object is localized relative to the other objects.

${ }^{2}$ Heading Error is the average of the signed pointing errors. Its value is close to $0^{\circ}$ when participants are oriented but it is randomly distributed when they are disoriented.
} 
objects in a distinct representation that was automatically updated with rotational movement. This is in line with arguments that spatial updating is limited to objects in one's immediate surroundings (Wang \& Spelke, 2000) that are maintained in a transient sensorimotor representation (Avraamides \& Kelly, 2008; Mou et al., 2004; Waller \& Hodgson, 2006).

Overall, the results from studies with nested environments suggest that people keep spatial information for each environment in separate representations. Relating information across representations, which presumably takes place when required by the task, may take place but at a considerable performance cost.

\subsection{Integration Assessed in between vs. within-Layout Judgments}

Another approach to examining whether people integrate locations from multiple layouts that they have encoded with temporal separation is to examine their judgments for spatial relations within the same layout vs. between layouts (e.g., Giudice, Klatzky, \& Loomis, 2009; Ishikawa \& Montello, 2006). If information in the two layouts is integrated into a single spatial representation at encoding then no performance differences are expected when comparing within- and between-layout judgments.

This paradigm has been used to investigate different types of layouts, from large scale environments in studies investigating navigation (Moar \& Carleton, 1982; Montello \& Pick, 1993; Golledge, Ruggles, Pellegrino \& Gale, 1993; Ishikawa et al., 2006) to table-top scenes (Giudice et al., 2009). However, findings from these studies are contradictory.

A number of studies indicate that even though participants are able to relate information derived from separate experiences by performing well above chance, they do better at within- than between-layout trials. For example, Montello and Pick (1993) had participants walk two routes within a building. Participants learned the two routes separately but they were then either verbally informed about the connection between the two routes, or experienced the relationship through navigation. In subsequent testing, participants pointed to non-visible landmarks on the two routes while walking in one of them. Although participants could point to objects in both routes with above chance accuracy, they did better when pointing to objects from the route they were travelling on than the other one. This pattern of results suggested that people did not integrate the spatial information from the two routes into a single representation. Instead, they computed intra-route information at the time of responding. This was also the case in a study by Ishikawa et al., (2006), where participants learned two separate routes by being driven along each route ten times. After the first three experiences a connecting path between the two routes was experienced in the learning routine. At the end of each learning experience participants had to estimate the direction and route distance between four landmarks that were previously experienced along the routes. After the fourth session, participants provided straight line distance and direction estimates between landmarks within and across routes. Participants' direction estimates across routes were above chance performance, although distance estimates did not differ significantly from guessing. Moreover, as participants gained more experience with the routes their 
performance improved. Thus, participants were able to relate to some degree the spatial information from the two layouts. But even so, the performance for between route landmarks was worse than within route landmarks suggesting that the two routes were not integrated into a single spatial representation in memory.

On the other hand, other studies have provided results compatible with integrating information from separate layouts into a single spatial representation (Holding \& Holding, 1989; Moar \& Carleton, 1982). For example, Moar and Carleton (1982) investigated whether people would integrate information from separately-learned, intersecting routes. Navigation was simulated by showing participants photographs taken from the routes. Participants then had to estimate the distance and direction between two locations presented in two slides on a screen that were either from the same route or from different routes. Participants performed comparably when providing estimates for within-route and cross-route pairs of places, suggesting that they were able to integrate the two routes into a single spatial representation.

A complicating factor from interpreting findings from studies comparing betweenlayout and within-layout judgments to determine whether single or distinct representations are maintained is that previous research has indicated that spatial performance is influenced by the temporal and spatial separation of locations (McNamara, Halpin, \& Hardy, 1992). Studies using within vs. between-layout judgments typically involve learning layouts of objects separately in time (e.g., Ishikawa et al., 2006; but see Greenauer \& Waller, 2010). Also, although some studies have controlled for spatial separation (e.g., Giudice et al., 2009; Montello \& Pick, 1993), others have not (e.g., Greenauer \& Waller, 2010). Furthermore, a study by Greenauer and Waller (2010) demonstrated that despite comparable performance for within- and between-layout judgments, layouts were maintained in distinct representations. In this study, participants studied objects placed in the center of a room forming two adjacent arrays. The two arrays were viewed simultaneously (Exp.1, 2, and 4) or sequentially (Exp.3) and were distinguished by colored disks. Importantly, each array had its own axis of bilateral symmetry which was misaligned with the learning view of the observer, and participants were instructed to learn the layouts along their symmetry axes. JRD responses for withinlayout trials indicated that each array was maintained in memory from a different preferred direction that was determined by its axis of bilateral symmetry. In contrast, between-layout responses were facilitated along the direction determined by the learning view of the participant. Thus, despite the similar performance in overall accuracy and latency in within- and between-layout trials, these findings suggest that, in line with theories of hierarchical encoding (Hirtle \& Jonides, 1985), the two layouts were organized around distinct microreference frames whose relation was specified by a more global macroreference frame.

One paradigm that controls for the spatial separation of layouts is the transfer of reference frames, which we present in the next section.

\subsection{The Transfer of Reference Frames across Layouts}

Kelly and McNamara (2010) developed a new method to study how people organize in memory distinct layouts that are learned in sequence (see also Kelly, Avraamides, \& McNamara, 2010). This method examines whether the reference frame that is used 
to organize the first studied layout is transferred to the encoding of the second layout. It should be noted that finding that a common reference frame is used to encode two layouts does not necessarily mean that the layouts have been integrated into a single representation. However, the opposite finding -- i.e., that each layout is associated with a distinct reference frame (e.g., Greenauer \& Waller, 2010)-- is hard to accommodate with a single representation account.

In one study Kelly and McNamara (2010) had participants study an external layout of 7 objects from one of two perspectives $\left(0^{\circ}\right.$ or $\left.135^{\circ}\right)$. Following learning, 7 new objects were added to the scene which participants studied from a fixed perspective $\left(135^{\circ}\right)$. Testing with JRD involving only within-layout locations revealed that performance for the second layout was facilitated for imagined perspectives aligned with the study viewpoint of the first layout $\left(0^{\circ}\right.$ or $135^{\circ}$ depending on condition). According to the authors, participants established a reference frame from the study viewpoint of the first layout and subsequently used it to encode the locations of the second. This is congruent with findings that reference frames established through vision can be later used to encode haptic locations (Kelly \& Avraamides, 2011) and vice-versa (Kelly, Avraamides, \& Giudice, 2011).

The studies on the transfer of reference frames control for spatial separation of objects by using overlapping layouts. However, as their primary goal was to assess the reference frames used for encoding each layout, they have not included any between-layout judgments. Thus, although their findings are compatible with a single-representation for distinct layouts, they are not conclusive. In the next section we present results from a new experiment aimed at assessing the transfer of reference frames for layouts learned from different perspectives, while also using both withinand between-layout judgments.

\subsection{Integration of Layouts Encoded from Distinct Perspectives}

We have conducted an experiment to examine whether spatial locations in the same physical space but experienced as separate layouts from different perspectives are integrated into a single representation (Adamou, 2011). Meilinger, Berthoz, and Wiener (2011) have also examined the integration of spatial information that was viewed from different perspectives. In their study, participants learned two spatial layouts each containing 3 locations by either viewing both layouts from the same standpoint, or by viewing the second layout upon walking to a different standpoint that was offset by $90^{\circ}$ from the first. Following learning, participants in both conditions were instructed to walk the shortest path that linked the 6 locations. Participants were capable of relating spatial information across experiences in order to compute a path: path-planning performance in both conditions was only $3.7 \%$ longer than the shortest possible path. Additionally, participants made more errors when walking to targets of the first layout. The authors interpreted this finding as evidence that the locations of the first layout were transformed to the reference frame of the second.

The path-walking task used by Meilinger et al. (2011) relies strongly on participants' actual orientation and may have encouraged participants to update 
spatial information during movement from the first standpoint to the other. Unlike Meilinger et al., we used JRD, an off-line test of spatial memory that is less dependent on the observer's actual orientation (see Avraamides \& Kelly, 2008 for a discussion). Additionally, in contrast to previous studies on the transfer of reference frames (Kelly et al., 2011, 2010), in this study participants were internal to the layout. Participants first learned a layout of four objects from one orientation $\left(0^{\circ}\right)$ while standing in the center of a featureless round room in virtual reality (Fig.1). Once they memorized the layout, the objects were removed and participants rotated to the left to study a second layout from a different orientation $\left(210^{\circ}\right)$. Following learning, participants moved to a different laboratory and carried out a series of JRD trials (i.e., "imagine facing $\mathrm{x}$, point to y") using a computerized pointer. Following the logic of previous studies (e.g., Ishikawa \& Montello, 2006), trials involved pairs of objects from the same or different layouts.

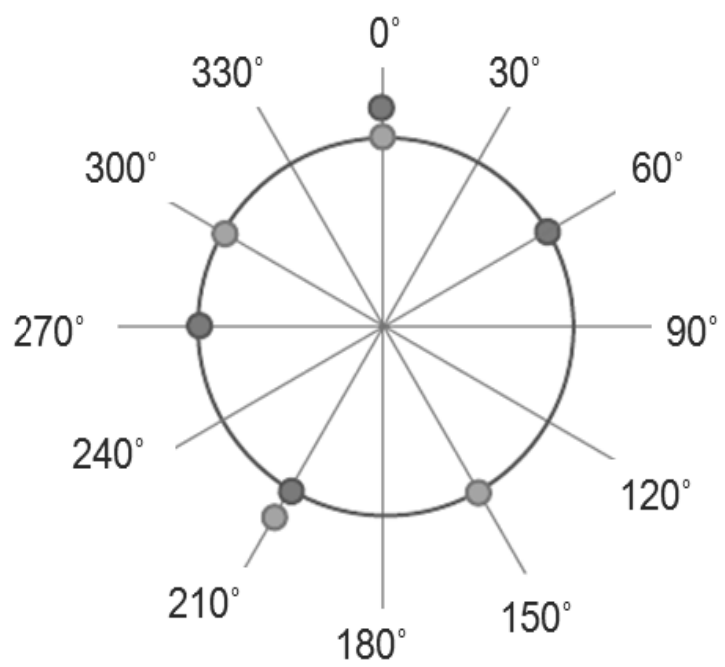

\section{Layout 1 objects, studied from $0^{\circ}$}

Layout 2 objects, studied from $210^{\circ}$

Fig. 1. A schematic illustration of the two spatial layouts. Participants studied one layout while facing $0^{\circ}$ and another while facing $210^{\circ}$. The assignment of layout identity ( 1 or 2 ) to learning order (first or second) was counterbalanced across participants. The study viewpoint was always $0^{\circ}$ for the layout studied first and $210^{\circ}$ for the layout studied second.

Results indicated that performance was above chance in between-layout trials confirming that participants were able to use information from the different layouts to carry out the task. However, they were considerably slower to respond in betweenlayout than within-layout trials suggesting that the two layouts were not integrated into a single spatial representation. Compatible with this conclusion were the analyses of latency to respond from imagined perspectives: for within-layout trials participants 


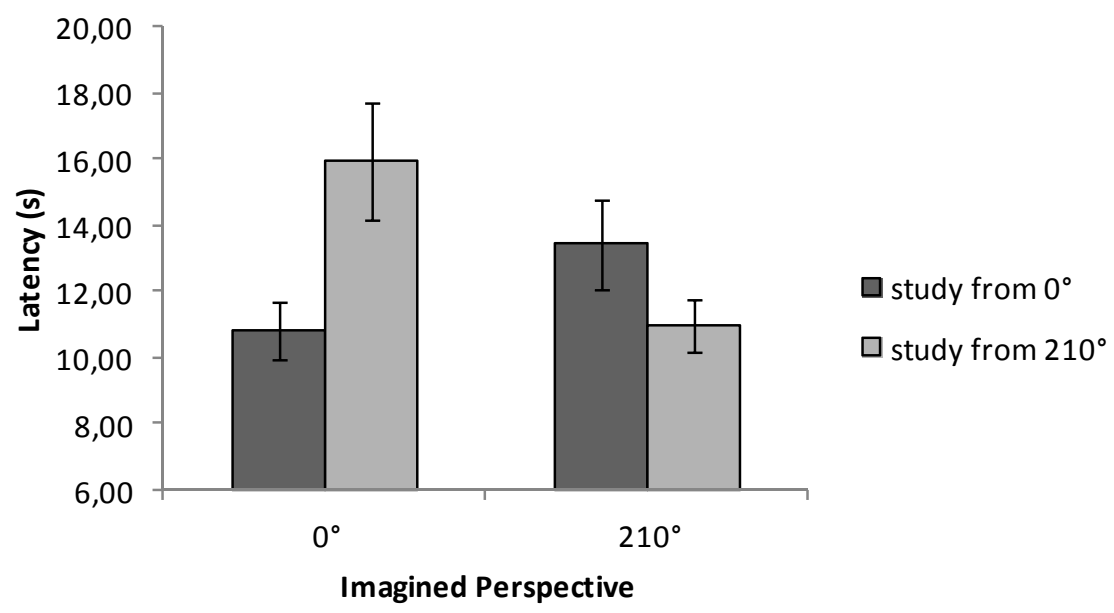

Fig. 2. Latency as a function of imagined perspective in within-layout trials. Only performance for the two viewpoints that were common in the two layouts is shown. Separate statistical analyses considering all imagined perspectives confirmed the presence of a preferred direction aligned with the study viewpoint of each layout.

were faster to respond from the perspective that was aligned with the study viewpoint of the layout $\left(0^{\circ}\right.$ or $210^{\circ}$ depending on learning order $)$ than the other imagined perspectives (Fig. 2).

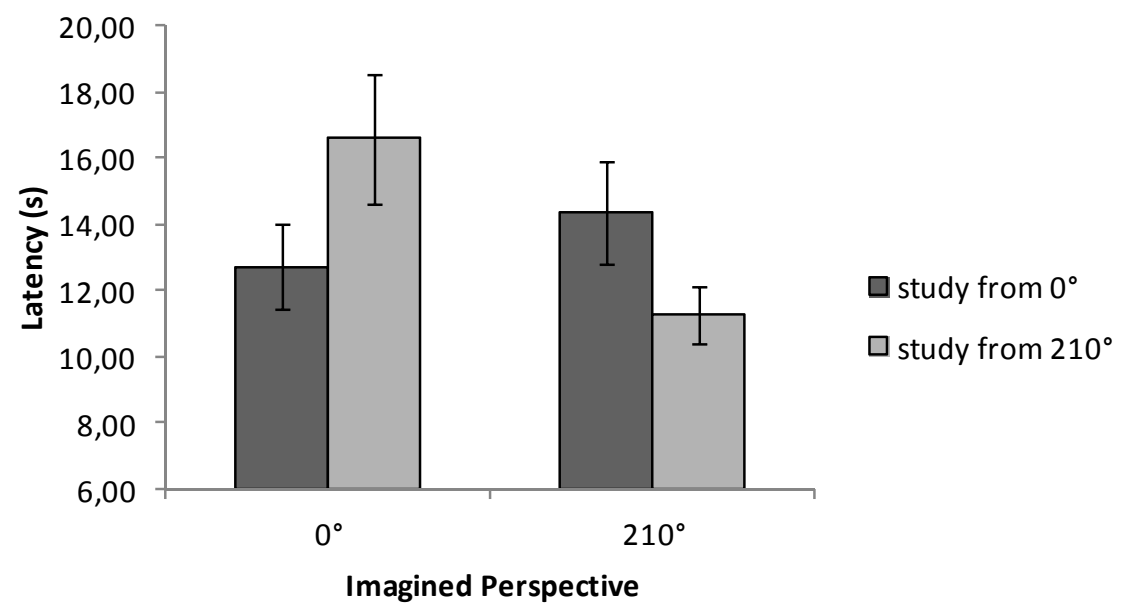

Fig. 3. Latency as a function of imagined perspective and the study viewpoint of the layout from which the orienting object was sampled in between-layout trials 
In between-layout trials, participants were faster to respond from imagined perspectives aligned with the study viewpoint of the layout from which the orienting object (i.e., the one defining the imagined perspective) came (Fig. 3).

The findings from this study indicate that when participants learn separate layouts of objects from different viewpoints, they keep these layouts in distinct spatial representations each with its preferred direction, even when the objects are dispersed within the boundaries of the same physical environment. This is compatible with the claims of Greenauer and Waller (2010) that layouts are maintained in distinct microreference frames. The transfer of reference frame observed in previous studies (e.g., Kelly \& McNamara, 2010) did not take place here, but numerous methodological differences could account for this failure to replicate. For example, the first layout was occluded when participants studied the second layout, and the circular room provided no global orientation cues. Further research is needed to determine the necessary conditions for reference frame transfer.

\section{Summary and Conclusions}

The findings from the studies we have reviewed here suggest that spatial locations encoded in memory as part of distinct perceptual experiences are kept in separate spatial representations. Each spatial representation can be organized around a different preferred direction on the basis of cues that are available during learning (Mou \& McNamara, 2002). People are flexible at picking up cues to establish a preferred direction and often transfer such cues from one experience to another (Kelly \& Avraamides, 2011). Furthermore, the spatial relation between two or more spatial representations is sometimes directly represented in memory (Greenauer \& Waller, 2010) and sometimes not (Wang \& Brockmole, 2003a).

Representing objects in memory in small clusters may be beneficial for everyday tasks that typically rely on processing only a small number of locations at a time. For example, on-line tasks such orienting ourselves in the local environment entail processing only a small number of immediate locations. Similarly, off-line tasks such as describing the layout of our house to a colleague who visits our office requires considering only the locations of the distal household objects and ignoring any objects in the immediate environment of the office. Clustering objects into small meaningful representations may thus allow us to activate only the spatial information that is needed at a given moment enabling us to operate within the capacity limits of working memory. Locations at our home can thus be clustered into smaller meaningful units (e.g., defined by rooms) that can be managed more easily during retrieval.

Although people may represent spatial information in appropriate, distinct representations, they are typically efficient at relating spatial information across representations. In the studies we have reviewed, participants could localize objects between layouts well above chance. Thus, when a task requires coordinating information across perceptual experiences, people seem capable of doing so at the time the information is needed (Meilinger et al., 2011). Although a performance cost 
is sometimes observed, this cost is by no means dramatic. In fact this cost may be modulated by a number of factors, such as the requirements of the task at hand. Different tasks place different demands on encoding and maintaining spatial relations among locations learned with extended spatial and temporal separation. For example, memorizing a route that one is travelling requires relating spatially the locations encountered on the route (e.g., landmarks, decision-points, etc) and storing these relations in memory. Indeed, neuroimaging studies employing subliminal priming methods have provided evidence that people encode and maintain in memory functional links between locations experienced along travelled routes (e.g., Janzen \& Westeijn, 2007; Shinazi \& Epstein, 2010). Thus, although people may normally default to maintaining separate representations for spatial representations derived from distinct perceptual experiences, they can integrate information into a single representation either at encoding or at a later stage if the task requires them to do so.

Acknowledgments. Support was provided by research grant OSSMA-206912 from the European Research Council to M.N.A.

\section{References}

1. Adamou, C.: Integration of visuo-spatial information encoded from different modalities. Unpublished MSc Thesis. University of Cyprus (2011)

2. Avraamides, M.N., Kelly, J.W.: Multiple systems of spatial memory and action. Cognitive Processing 9, 93-106 (2008)

3. Avraamides, M.N., Loomis, J.M., Klatzky, R.L., Golledge, R.G.: Functional equivalence of spatial representations derived from vision and language: evidence from allocentric judgments. Journal of Experimental Psychology. Learning, Memory, and Cognition 30, 804-814 (2004)

4. Giudice, N.A., Klatzky, R.L., Loomis, J.M.: Evidence for amodal representations after bimodal learning: integration of haptic-visual layouts into a common spatial image. Spatial Cognition \& Computation 9, 287-304 (2009)

5. Golledge, R.G., Ruggles, A.J., Pellegrino, J.W., Gale, N.D.: Integrating route knowledge in an unfamiliar neighborhood: Along and across route experiments. Journal of Environmental Psychology 13, 293-307 (1993)

6. Greenauer, N., Waller, D.: Intrinsic array structure is neither necessary nor sufficient for nonegocentric coding of spatial layouts. Psychonomic Bulletin \& Review 15, 1015-1021 (2008)

7. Greenauer, N., Waller, D.: Micro- and macroreference frames: Specifying the relations between spatial categories in memory. Journal of Experimental Psychology. Learning, Memory, and Cognition 36, 938-957 (2010)

8. Hirtle, S.C., Jonides, J.: Evidence of hierarchies in cognitive maps. Memory \& Cognition 13, 208-217 (1985)

9. Hodgson, E., Waller, D.: Lack of set size effects in spatial updating: Evidence for offline updating. Journal of Experimental Psychology. Learning, Memory, and Cognition 32, 854-866 (2006)

10. Holding, C.S., Holding, D.H.: Acquisition of route network knowledge by males and females. Journal of General Psychology 116, 29-41 (1989) 
11. Ishikawa, T., Montello, D.R.: Spatial knowledge acquisition from direct experience in the environment: individual differences in the development of metric knowledge and the integration of separately learned places. Cognitive Psychology 52, 93-129 (2006)

12. Janzen, G., Weststeijn, C.G.: Neural representation of object location and route direction: an event-related fMRI study. Brain Research 1165, 116-125 (2007)

13. Kelly, J.W., Avraamides, M.N., Loomis, J.M.: Sensorimotor alignment effects in the learning environment and in novel environments. Journal of Experimental Psychology. Learning, Memory, and Cognition 33, 1092-1107 (2007)

14. Kelly, J.W., Avraamides, M.N.: Cross-sensory transfer of reference frames in spatial memory. Cognition 118, 444-450 (2011)

15. Kelly, J.W., McNamara, T.P.: Reference frames during the acquisition and development of spatial memories. Cognition 116, 409-420 (2010)

16. Kelly, J.W., Avraamides, M.N., Giudice, N.A.: Haptic experiences influence visually acquired memories: reference frames during multimodal spatial learning. Psychonomic Bulletin \& Review 18, 1119-1125 (2011)

17. Kelly, J.W., Avraamides, M.N., McNamara, T.P.: Reference Frames Influence Spatial Memory Development within and Across Sensory Modalities. In: Hölscher, C., Shipley, T.F., Olivetti Belardinelli, M., Bateman, J.A., Newcombe, N.S. (eds.) Spatial Cognition VII. LNCS (LNAI), vol. 6222, pp. 222-233. Springer, Heidelberg (2010)

18. McNamara, T.P., Halpin, J.A., Hardy, J.K.: Spatial and temporal contributions to the structure of spatial memory. Journal of Experimental Psychology. Learning, Memory, and Cognition 18, 555-564 (1992)

19. McNamara, T.P.: How Are the Locations of Objects in the Environment Represented in Memory? In: Freksa, C., Brauer, W., Habel, C., Wender, K.F. (eds.) Spatial Cognition III. LNCS (LNAI), vol. 2685, pp. 174-191. Springer, Heidelberg (2003)

20. McNamara, T.P., Rump, B., Werner, S.: Egocentric and geocentric frames of reference in memory of large-scale space. Psychonomic Bulletin \& Review 10, 589-595 (2003)

21. Meilinger, T., Berthoz, A., Wiener, J.M.: The integration of spatial information across different viewpoints. Memory \& Cognition 39, 1042-1054 (2011)

22. Mou, W., McNamara, T.P.: Intrinsic frames of reference in spatial memory. Journal of Experimental Psychology. Learning, Memory, and Cognition 28, 162-170 (2002)

23. Moar, I., Carleton, L.R.: Memory for routes. Quarterly Journal of Experimental Psychology 34, 381-394 (1982)

24. Montello, D.R., Pick, H.L.: Integrating Knowledge of Vertically Aligned Large-Scale Spaces. Environment and Behavior 25, 457-484 (1993)

25. Mou, W., McNamara, T.P., Valiquette, C.M., Rump, B.: Allocentric and egocentric updating of spatial memories. Journal of Experimental Psychology. Learning, Memory, and Cognition 30, 142-157 (2004)

26. Mou, W., Zhao, M., McNamara, T.P.: Layout geometry in the selection of intrinsic frames of reference from multiple viewpoints. Journal of Experimental Psychology. Learning, Memory, and Cognition 33, 145-154 (2007)

27. Presson, C.C., DeLange, N., Hazelrigg, M.D.: Orientation-specificity in kinesthetic spatial learning: the role of multiple orientations. Memory \& Cognition 15, 225-229 (1987)

28. Presson, C.C., DeLange, N., Hazelrigg, M.D.: Orientation specificity in spatial memory: what makes a path different from a map of the path? Journal of Experimental Psychology. Learning, Memory, and Cognition 15, 887-897 (1989)

29. Roskos-Ewoldsen, B., McNamara, T.P., Shelton, A.L., Carr, W.: Mental representations of large and small spatial layouts are orientation dependent. Journal of Experimental Psychology. Learning, Memory, and Cognition 24, 215-226 (1998) 
30. Schinazi, V.R., Epstein, R.A.: Neural correlates of real-world route learning. NeuroImage 53, 725-735 (2010)

31. Shelton, A.L., McNamara, T.P.: Multiple views of spatial memory. Psychonomic Bulletin \& Review 4, 102-106 (1997)

32. Shelton, A.L., McNamara, T.P.: Visual memories from nonvisual experiences. Psychological Science 12, 343-347 (2001)

33. Werner, S., Schmidt, K.: Environmental reference systems for large-scale. Spatial Cognition and Computation 1, 447-473 (2000)

34. Waller, D., Hodgson, E.: Transient and enduring spatial representations under disorientation and self-rotation. Journal of Experimental Psychology. Learning, Memory, and Cognition 32, 867-882 (2006)

35. Waller, D., Montello, D.R., Richardson, A.E., Hegarty, M.: Orientation specificity and spatial updating of memories for layouts. Journal of Experimental Psychology: Learning, Memory, and Cognition 28, 1051-1063 (2002)

36. Wang, R.F., Brockmole, J.R.: Human navigation in nested environments. Journal of Experimental Psychology. Learning, Memory, and Cognition 29, 398-404 (2003a)

37. Wang, R.F., Brockmole, J.R.: Simultaneous spatial updating in nested environments. Psychonomic Bulletin \& Review 10, 981-986 (2003b)

38. Wang, R.F., Spelke, E.S.: Updating egocentric representations in human navigation. Cognition 77, 215-250 (2000) 